Article

\title{
The Temporal PET Camera: A New Concept With High Spatial and Timing Resolution for PET Imaging
}

\author{
Alain Iltis ${ }^{1, *}$ and Hichem Snoussi ${ }^{2}$ \\ ${ }^{1}$ Damavan Imaging, Technopole de l'Aube, 2 rue Gustave Eiffel, 10000 Troyes, France \\ ${ }^{2}$ University of Technology of Troyes Institute Charles Delaunay, UMR CNRS 6279 12, rue Marie \\ Curie, 10010 Troyes, France; E-Mail: hichem.snoussi@utt.fr \\ * Author to whom correspondence should be addressed; E-Mail: damavan.imaging@gmail.com; \\ Tel.: 0033-3-2571-8087.
}

Academic Editor: Gonzalo Pajares Martinsanz

Received: 14 April 2015 / Accepted: 23 July 2015 / Published: 6 August 2015

\begin{abstract}
This contribution proposes a new temporal PET camera concept yielding a precise spatio-temporal localization of a scintillation event within a monolithic scintillator. This concept is promising for PET imaging. The key idea behind this concept is the ability of the system to accurately localize the region of detected un-scattered photons on the Si-PMT detector plane. Then, by ray tracing, an accurate estimate of the depth and timing of the scintillation event is provided. An estimation of the potential performance of such a system, based on extensive Monte Carlo simulations, is also presented.
\end{abstract}

Keywords: Gamma camera; Si-PMT, PET imaging; TOF; CRT; Spatial resolution; monolithic scintillators

\section{Introduction}

The use of monolithic scintillator-based detectors is promising for designing time of flight (TOF) positron electron tomography (PET) devices. Monolithic scintillators exhibit a number of interesting properties such as excellent energy resolution, high $\gamma$ photon capture efficiency and relatively simple detector assembly. This detector concept is all the more interesting with the availability of Si-PMT which offers high gain, fast response, insensitivity to magnetic fields and potential cost effectiveness in a compact package. 
Yet the concepts so far proposed to recover the localization in space (3D position $\mathrm{X}, \mathrm{Y}, \mathrm{Z}$ ) and time $T_{0}$ of the scintillation event occurring in a monolithic scintillator suffer from a number of limitations, such as:

- If an Anger logic is used to recover the spatial localization of the scintillation event (projection of the 3D position on the (X,Y)-plane), the crystal must be thin (around $10 \mathrm{~mm}$ ) to have good spatial resolution. However, in that case, many events could be lost due to insufficient thickness to absorb the $511 \mathrm{keV} \gamma$ ray.

- Uncertainties on scintillation light ray tracing related to the difference of propagation speed between UV photons $(n=1,9)$ and $\gamma$ photons $(n=1)$ do not allow very precise timing of the scintillation event (estimation of the initial time $T_{0}$ ).

- Obtaining information about the interaction depth requires either two layers of crystals or two layers of photo-detectors, which raises the cost of the system.

Recently, very promising results have been obtained applying an Si-PMT array coupled to a bare $18 \mathrm{~mm} \times 16 \mathrm{~mm} \times 10 \mathrm{~mm}$ monolithic $\mathrm{LaBr}_{3}: 5 \% \mathrm{Ce}$ [1]. A spatial resolution of $1.6 \mathrm{~mm}$ FWHM was obtained. The timing resolution was $C R T=198 \mathrm{ps}$. The front side read-out necessary for this geometry was described as tricky.

In this work, we propose a new concept yielding a precise localization of a scintillation event in space and time with a monolithic scintillator. This concept will be analyzed here for the case of PET (511 keV $\gamma$ ), but it can also be used at other energies as long as the incidence angle of the $\gamma$ ray can be constrained (Lead collimators for SPECT, Compton camera, calorimeters, ....). Our objective, in this paper, is to describe the proposed concept and estimate its ultimate precision performances in an idealized case for PET applications. The paper is organized as follows. Section 2 introduces the concept of a Gamma camera and some recent works aimed at enhancing its performances. Section 3 is dedicated to the major contribution of this paper which is the new concept of temporal Gamma cameras. Synthetic simulations as well as performance evaluation of the spatio-temporal localization of the temporal Gamma camera are introduced in this section. Section 4 concludes the paper.

\section{The Gamma Camera Concept}

In the Anger $\gamma$ camera, the $\gamma$ ray incidence direction is controlled through absorption on a lead collimator, so that the photons' incidence is close to normal on the scintillator plane. Absorbtion of the $\gamma$ ray creates a scintillation event. The UV scintillation light is emitted isotropically. The light is then channelled through the plane by reflection on the interfaces. The number of photons detected on the scintillation plane roughly decreases by $1 / R$, where $R$ is the distance to the projection of the scintillation point on the detector plane. This property is used to find the center of the distribution of photons and thus gives the X,Y coordinates of the scintillation event [2]. However, there are still a number of drawbacks with this method:

- The photon distribution is spread out, so the images are noisy.

- The reconstruction does not work well close to the edges of the plates, so it is not possible to tile many plates to make a bigger one. The crystals must be big enough, restricting the choice of available scintillators to NaI:Tl and CsI:Tl. 
- This method has a severe limitation taking into account events which are close in space and time: the pile up effect [6].

- The scintillator energy resolution is a key item in obtaining good images with an Anger camera, so experiments have been done using $\mathrm{LaBr} 3: 5 \% \mathrm{Ce}$ [3]. Sub-millimetric spatial resolution has been obtained on a special geometry at $140 \mathrm{keV}$. However, the conditions of the experiment were far from the actual setup in radiology.

- $\mathrm{NaI}(\mathrm{Tl})$ Gamma cameras have been used in the past for PET [7,10]. However, the localization of the events was too coarse. In addition, the stopping power of $\mathrm{NaI}(\mathrm{Tl})$ was too low and the Compton effect was an issue. The concept we propose below uses a denser crystal than $\mathrm{NaI}(\mathrm{Tl})$ and should overcome most of the drawbacks of previous Gamma camera systems. It also should be significantly cheaper to produce than conventional lutetium oxyorthosilicate (LSO)-based PET.

\section{Proposed Temporal Gamma Camera}

The availability of fast, efficient, segmented detectors like Si-PMT, fast scintillators like LaBr3:Ce and very fast mixed ASICs [5], able to give a read-out and to trigger 16 channels in less than 100 ps could open the way to using temporal information rather than photon counts to reconstruct the scintillation event. But is there any temporal information that could be used for image reconstruction?

We consider a monolithic scintillator crystal of thickness $h$, with a similar design to a state-of-the-art Gamma camera. The upper surface would be roughened and covered by a white wrapping so as to ensure a diffusive surface. The lower surface would be polished with a layer of segmented Si-PMT glued to the crystal. In this paper, we will not consider the impact on resolution of Si-PMT segmentation pitch. No correction will be done for the photon detection efficiency of the Si-PMT. Once a photoelectric event $E(X, Y, Z, T)$ takes place inside the crystal at position $(X, Y, Z)$ and time $T$, the scintillation light is emitted isotropically following the scintillator emission light curve. Below, for the sake of clarity, we will discuss only the case of a pure photo-electric event. A future paper will discuss the impact of the Compton effect, but as forward scattering is dominant at $511 \mathrm{keV}$, Compton diffusion does not significantly affect the result. The photons directed towards the base of the crystal (see Figure 1) are not scattered and are detected directly. Their path is a straight line to the detector. All the other photons will be subjected to at least one scatter and thus have a longer light path in the crystal, subsequently impacting the photo-detectors.

Between the crystal and the detector, an optical interface with an index step is deployed. Thus, there is a critical angle $\theta_{c}$ above which the photon will be reflected and thus not detected. The unscattered photons will thus be located in a cone whereby the summit is the event position and the opening angle is $\theta_{c}$. If $n_{c}$ is the crystal index and $n_{g}$ is the index of the glue between the crystal and the Si-PMT, the critical angle is $\theta_{c}=\arcsin \left(n_{g} / n_{c}\right)$. Hence the image of the un-scattered photons on the detector plane will be a disc centered on the location of the interaction $(X, Y)$ and whose diameter will be $(L-Z) * \tan \left(\theta_{c}\right)$. We can label those un-scattered photons by order of detection on the detector plane, where $P_{n}$ will denote the $n^{t h}$ photon detected with spatio-temporal coordinates on the detector plane $\left(x_{n}, y_{n}, t_{n}\right)$. Figure 1 illustrates the behavior of emitted photons after the scintillation event: (a) photons emitted inside the cone are detected directly by the photodetector, (b) photons emitted outside the cone in the downside 
direction are reflected before being scattered by the upper plane of the crystal, and (c) photons emitted in the upper direction will be scattered in a random direction. Scattering of scintillation photons could also occur in some inclusions inside the crystal. This would create secondary centers of emission in the crystal, mimicking a Compton event. However, both $\mathrm{CeBr} 3$ and LYSO are available on the market and have the advantageous property of a very low concentration of scattering imperfections (inclusion is of a size less than $1 \mathrm{~mm}$ with a density less than $0.1 \mathrm{~cm}^{-3}$ ). Hence, the number of scattered photons, after the first scintillation event, will be below the detection limits in actual systems.

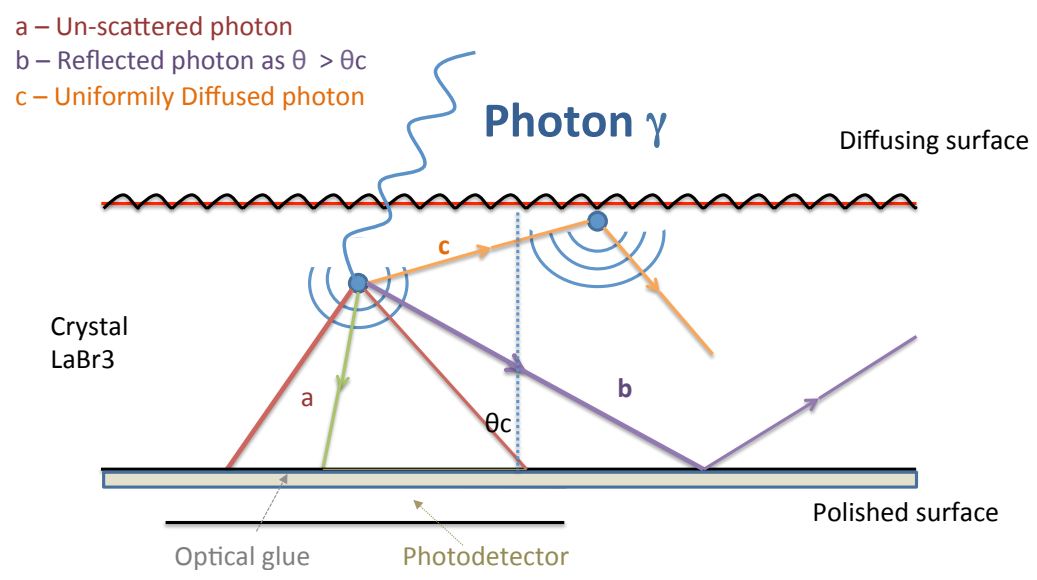

Figure 1. Illustration of the different behaviors of emitted photons before detection on the Si-PMT: (a) the photons inside the cone are detected; (b) Photons outside the cone and in the downside direction are reflected before being scattered by the diffusive surface and (c) the photons emitted in the upside direction are uniformly scattered when they reach the diffusing surface.

The rationale behind the proposed technique is the possibility of accurately estimating the center and the radius of the image disc formed by the proportion of un-scattered photons among the flood of photons emitted by the scintillation event. The detection process is illustrated in Figure 1 and detailed as follows:

- The first detected photons, during the characteristic transit time inside the crystal $\left(T_{\text {transit }} \approx h * n_{c} / c\right)$, will be those which are not scattered.

- The radius $r$ of the disc where photons are detected will grow over time until the critical angle $\theta_{c}$ is reached.

- After the characteristic transit time, the first scattered photons (by the upper surface) will reach the detector plane. However, as their angle of incidence is randomized by the scattering event, the scattered photons are spread over a much wider zone than the non-scattered photons. Hence, even a long period after the scintillation event, the un-scattered event disc will appear to be much denser than the whole spatial distribution.

Consequently, if the detector plane is equipped with segmented detectors able to register the time of photon arrival with a time precision better than $h * n_{c} / c$, it should be possible to reconstruct a temporal image $\left(x_{n}, y_{n}, t_{n}\right)$ of the $n$ first photons emitted by the scintillation event. This spatio-temporal image will allow: 
- Estimating the center and the diameter of the disc of un-scattered photons. The spatial localization $(X, Y, Z)$ of the scintillation event could then be deduced.

- More interestingly, by putting a high statistical weight on the first photons, a ray tracing allows us to better constrain $(X, Y)$ and to accurately time the scintillation event $(T)$.

More details on the spatio-temporal localization of the scintillation event from the images $\left(x_{n}, y_{n}, t_{n}\right)$ will be given in subsection 3.2.

\subsection{Simulation of the Images on the Detector Planes}

The objective of this subsection is to simulate the spatio-temporal images obtained on the detector plane after a $511 \mathrm{keV}$ photo-electric event. The crystal will be $\mathrm{LaBr} 3: 5 \% \mathrm{Ce}$. Its properties are taken from [4]. This crystal is selected because it has the highest light field of the commercially available crystals in the emission's first nanosecond. This property is the key success factor for this kind of imagery. As a PET system has been designed by the University of Pennsylvania with LaBr3:Ce [7], we believe its hygroscopy could be handled. If it is not the case, the concept below would still work with LSO:Ce or LYSO:Ce. The index of the crystal is $n_{c}=1.9$ for the $380 \mathrm{~nm}$ maximum emission. Considering the crystals available for lutetium silicates and $\mathrm{LaBr} 3$, the plate described here could have the following dimensions: $100 \mathrm{~mm} \times 250 \mathrm{~mm} \times 30 \mathrm{~mm}$. The crystal has a thickness of $30 \mathrm{~mm}$. The entrance face of the $\gamma$ photons is rough and is covered with a white reflector. We will consider this surface as perfectly diffuse in the simulations below (i.e., the photons striking it are scattered in all directions with an equal probability). The bottom face is polished. Si-PMT is glued to this face with an optical grease with an index of $n_{g}=1.4$. The critical angle is then $\theta_{c}=\arcsin \left(n_{g} / n_{c}\right)=47.4^{\circ}$ and the transit time is $T_{\text {transit }}=h * n_{c} / c=190 \mathrm{ps}$.

A $511 \mathrm{keV} \gamma$ photon enters the crystal and is subject to a photo-electric event located at $\left(X_{0}, Y_{0}, Z_{0}, T_{0}\right)$. The entrance face is chosen as the reference plane $(Z=0)$. The rise time of this crystal is taken as 750 ps. The UV photons emitted by the photoelectric event are emitted randomly in all 3D directions ( $4 \pi$ steradian). The emission law $N(t)$ is assumed to be a piecewise function composed of a non-decreasing part between $t=0$ and $t_{\text {rise }}=750 \mathrm{ps}$ and a decreasing function between $t_{\text {rise }}$ and $t=+\infty$ with a decay time $\tau_{2}=16 n s$ as follows:

$$
\left\{\begin{array}{l}
N(t)=B\left(1-e^{-t / \tau_{1}}\right) \text { for } 0 \leq t \leq 750 \mathrm{ps} \\
N(t)=A e^{-\left(t-t_{\text {rise }}\right) / \tau_{2}} \text { for } \quad t \geq 750 \mathrm{ps}
\end{array}\right.
$$

where the parameters $A, B$ and $\tau_{1}$ are fixed so that the continuity at the time $t=750 \mathrm{ps}$ is ensured and so that the total number of emitted photons is equal to 32700 for the $511 \mathrm{keV} \gamma$ photon:

$$
\int_{t=0}^{+\infty} N(t) d t=32700
$$

In order to illustrate the temporal behavior of the density of detected un-scattered photons inside the critical disc $\mathcal{D}_{c}$ (intersection of the critical cone and the detection plane: in green on figures), the images $\left(x_{n}, y_{n}, t_{n}\right)$ of detected photons are plotted in Figure 2 for $Z_{0}=5 \mathrm{~mm}$ and different selected time shots (at $t=274 \mathrm{ps}$ (100 ps after the detection of the first photon), around the rising time at $t_{\text {rise }}=774 \mathrm{ps}$ 
and around $3 \mathrm{~ns}$ ). It can be noted that, at the beginning, the first detected photons are inside the critical $\operatorname{disc} \mathcal{D}$. Then, the density of photons inside the critical disc increases and a few photons start to appear outside the disc. This behavior is propagated through time: the scattered photons populate the region outside the critical disc, but this later remains highly dense. Note that around the rise time $t_{\text {rise }}=750$ ps, the disc is well filled and only a few photons are outside it. This property will be exploited in the next subsection in order to yield a robust and accurate spatio-temporal localization of the photo-electric event. To further illustrate this property, the evolution through time of the number of detected photons inside and outside the critical disc, as well as their proportion, are reported in Figure 3a,b respectively. It is worth noting from Figure 3 that, until $1 \mathrm{~ns}$, at least $95 \%$ of photons are detected inside the critical $\operatorname{disc} \mathcal{D}$.
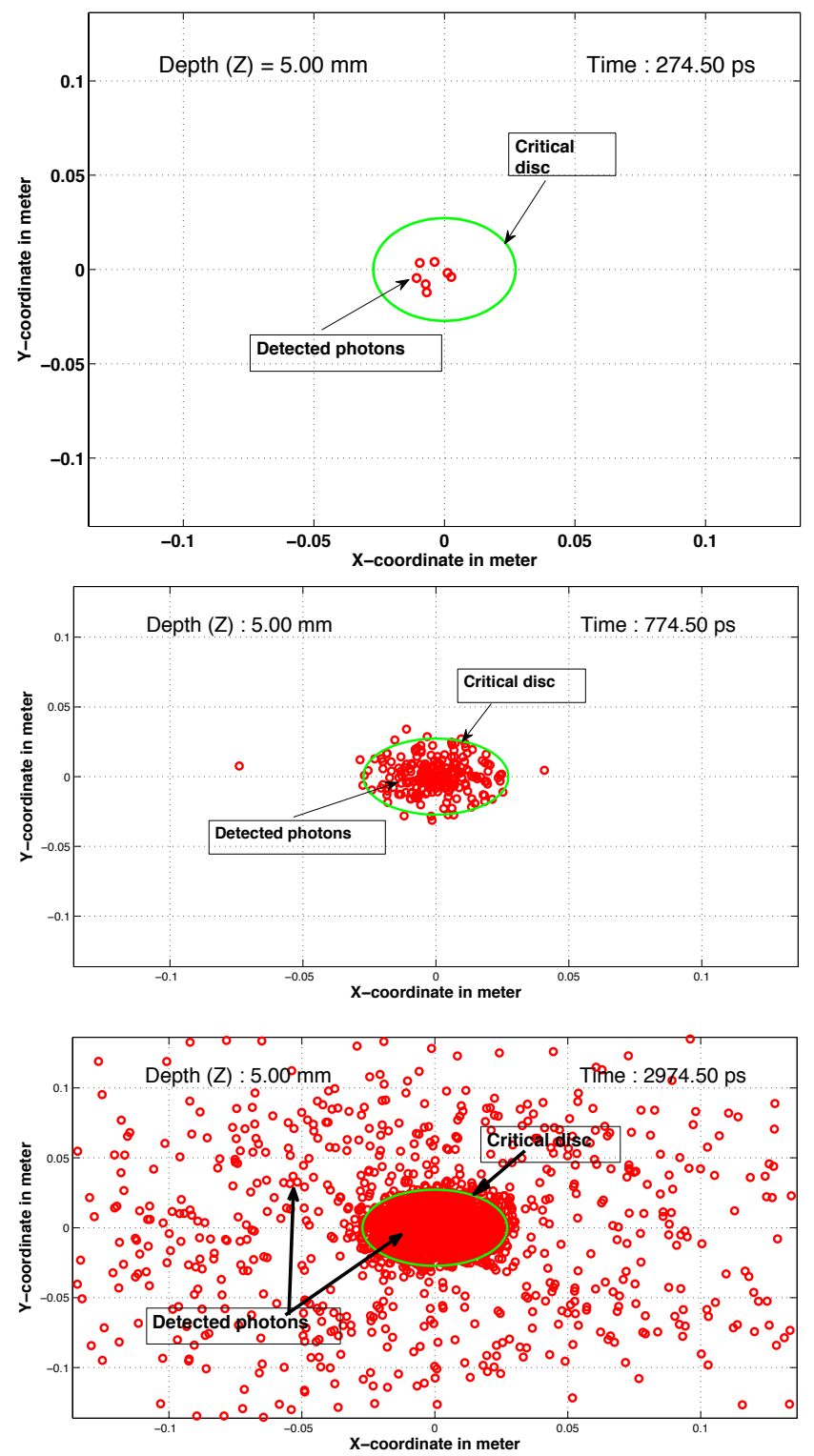

Figure 2. Images at different time shots for depth $\mathrm{Z}=5 \mathrm{~mm}$. 


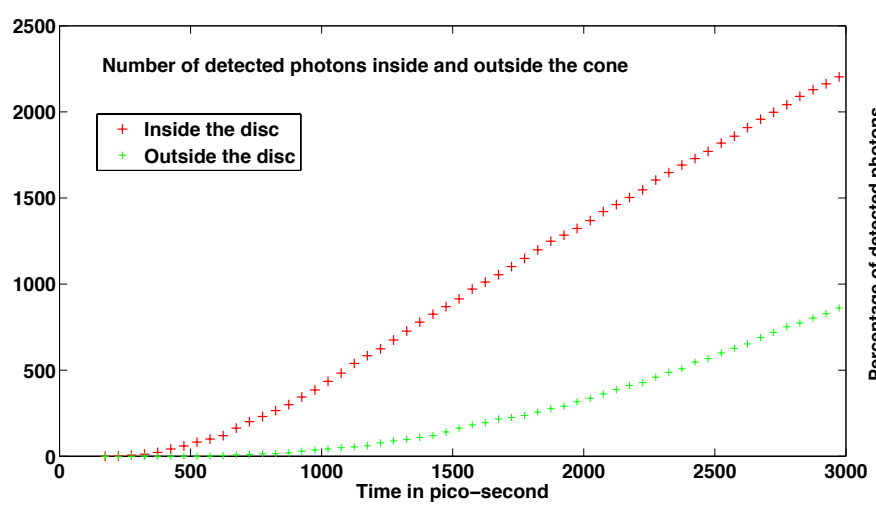

(a)

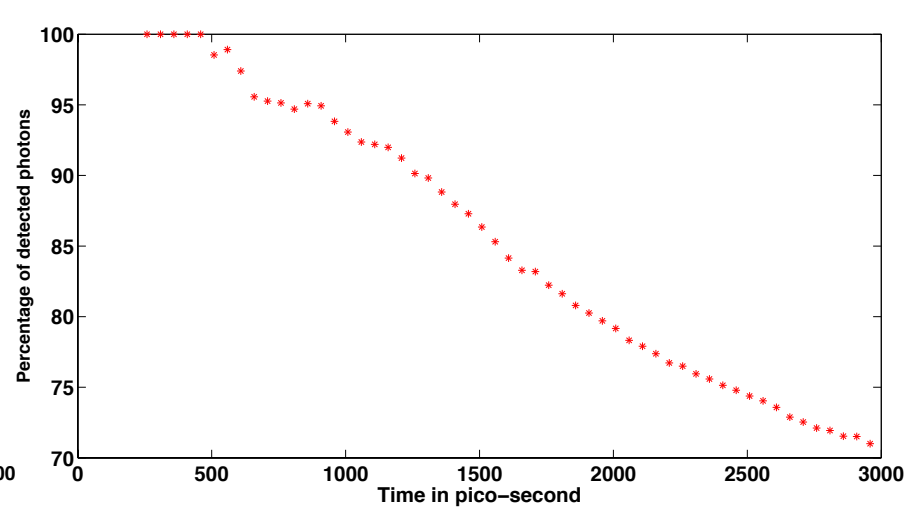

(b)

Figure 3. (a) Number of detected photons inside and outside the critical disc; (b) Percentage of detected photons inside the critical disc.

Figure 4 illustrates the temporal images $\left(x_{n}, y_{n}, t_{n}\right)$ for each of those different depths $Z_{0}=10 \mathrm{~mm}$, $Z_{0}=15 \mathrm{~mm}, Z_{0}=20 \mathrm{~mm}$ and $Z_{0}=25 \mathrm{~mm}$. Note that as the depth $(Z)$ increases, the critical disc gets smaller and more photons are detected outside for the same time shot.

We will briefly discuss now what happens if the absorption of the energy of the Gamma photon is incomplete in the first event, and if a significant Compton scattering is present. The Klein-Nishina formula [13] shows that for $511 \mathrm{keV}$ photons, most of the energy is partitioned in the forward scattering sector (i.e., $\theta<\theta_{c}$ ). If the scattered photon is diffused at an angle lower than $\theta_{c}$, the cone of un-scattered photons emitted in case of a photoelectric event will be confined inside the cone of the first event. Thus, all Compton photons scattered at an angle lower than $\theta_{c}$ will not significantly impact the precision of locating the interaction.

\subsection{Estimation of the Spatio-Temporal Localization of the Scintillation Event}

The critical disc $\mathcal{D}$ has the useful property of being quickly filled by the un-scattered photons. The scattered photons may later be detected outside $\mathcal{D}$, which remains, however, highly dense compared to the whole detection plan. Using this property, the 2D spatial localization process consists of first estimating the ellipse containing $95 \%$ of detected photons. The ellipse parameters yield an estimate of the barycenter $\left(\hat{X}_{0}, \hat{Y}_{0}\right)$ and an estimate of the radius $\hat{r}_{c}$ of the critical disc. As the critical angle $\theta_{c}$ is assumed to be known, the estimate $\hat{Z}_{0}$ can be deduced by the following relation:

$$
\hat{Z}_{0}=L-\hat{r}_{c} /\left(\tan \left(\theta_{c}\right)\right.
$$

By ray tracing, an estimate of the scintillation time could, in turn, be deduced by considering the time $T_{1}$ of the first detected photon at position $\left(X_{1}, Y_{1}\right)$. In fact, the trajectory length of the first detected photon is estimated as:

$$
\hat{D}_{1}=\sqrt{\left.\left(L-\hat{Z}_{0}\right)^{2}+\left(\left(X_{1}-\hat{X}_{0}\right)^{2}+\left(Y_{1}-\hat{Y}_{0}\right)^{2}\right)\right)}
$$

Then, an estimate of the scintillation time $T_{0}$ could be deduced as follows:

$$
\hat{T}_{0}=T_{1}-\hat{D}_{1} n_{c} / c
$$

where $c / n_{c}$ is the photon speed inside the crystal. 

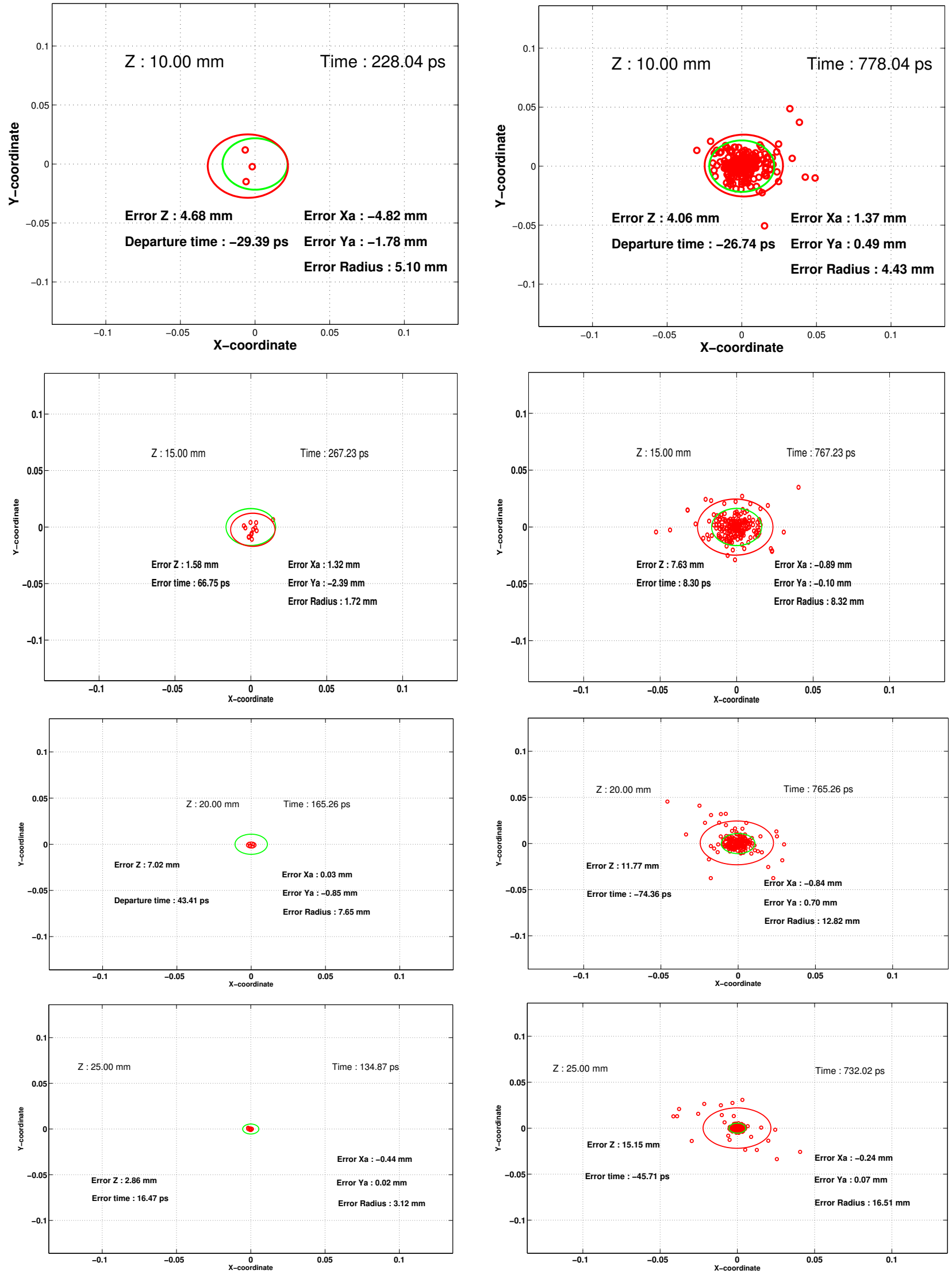

Figure 4. Images at different time shots and different depths. From top to bottom: $Z=10 \mathrm{~mm}, Z=15 \mathrm{~mm}, Z=20 \mathrm{~mm}$ and $Z=25 \mathrm{~mm}$. 
The estimation of the spatio-temporal localization $\left(\hat{X}_{0}, \hat{Y}_{0}, \hat{Z}_{0}, \hat{T}_{0}\right)$ of the scintillation event could be done at any time after the detection of the first incident photon. However, the performance of this estimation varies according to the time chosen for computing the estimates. In fact, the number of detected photons and their spatial distribution evolve in time: at the beginning, only the critical disc is populated by un-scattered photons, but the number of photons remains low to provide a (statistically) robust estimate of the spatio-temporal localization. On the other hand, as the number of detected photons increases with time, the estimation of the ellipse enclosing $95 \%$ of the points becomes less efficient. In order to illustrate this statement, the absolute error of the $\left(X_{0}, Y_{0}, Z_{0}, T_{0}\right)$ estimates are plotted in Figure 5a-d, respectively, for the case of depth $Z=5 \mathrm{~mm}$. Note from Figure 5a,b, that the estimates of $X_{0}$ and $Y_{0}$ are very accurate with a minimum of $3 \times 10^{-2} \mathrm{~mm}$ error for $X$ and $9 \times 10^{-4} \mathrm{~mm}$ error for $Y$. Also, note the fact that the performance of this estimation does not yield a regular feature through time. However, it can be seen, from Figure $5 \mathrm{c}$,d, that the precision of the $Z_{0}$ and $T_{0}$ estimates decreases monotonically through time. This is due to the fact that the estimate of the disc radius is less accurate over time. A minimum error of $1 \mathrm{~mm}$ for $Z$ and $6.5 \mathrm{ps}$ for $T$ could be achieved around the rising time of 750 ps. Figure 6 contains the same images as Figure 2 for 3 selected time shots, but with showing the estimation results and plotting the estimate of the ellipse (approximating the critical disc).

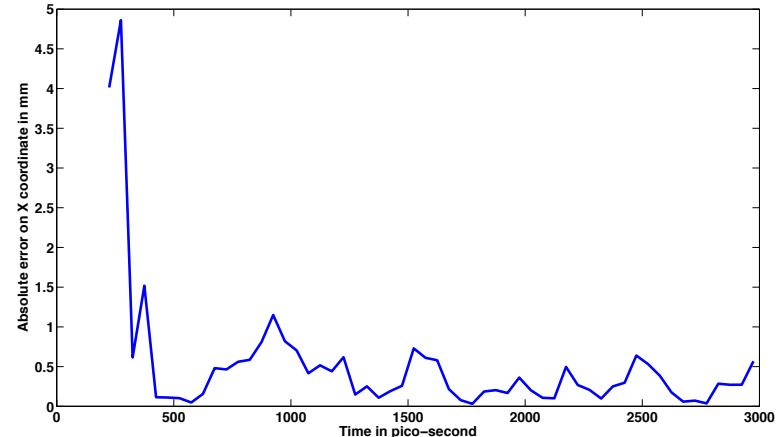

(a)

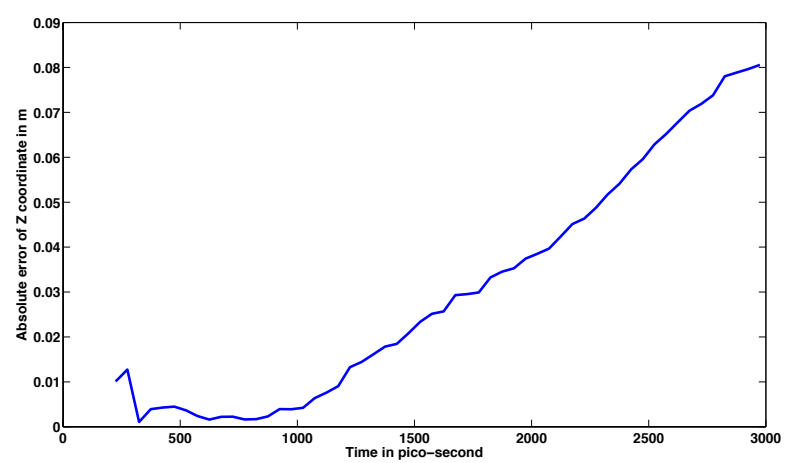

(c)

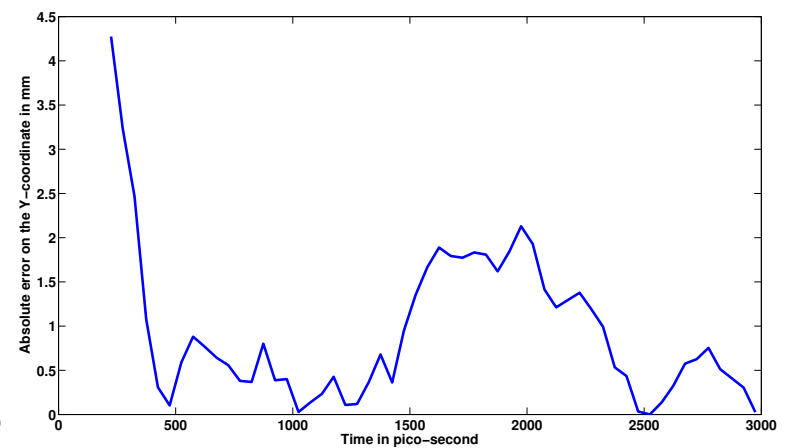

(b)

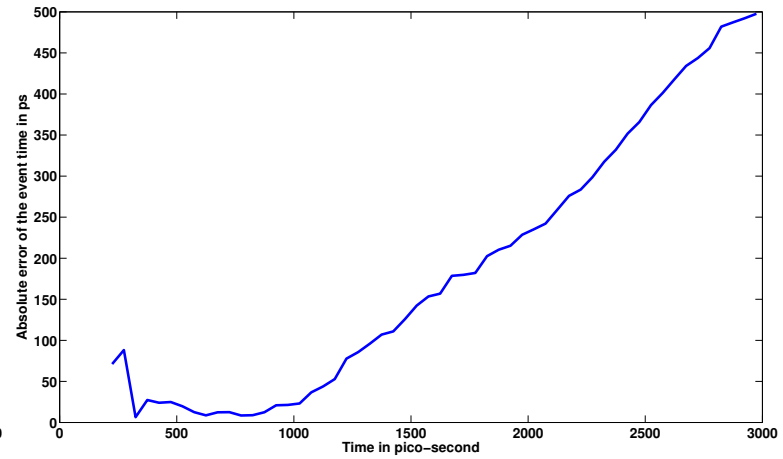

(d)

Figure 5. (a) Absolute error of the $X$ estimate; (b) Absolute error of the $Y$ estimate; (c) Absolute error of the $\mathrm{Z}$ estimate and (d) Absolute error of the $\mathrm{T}$ estimate.

In order to confirm the precision of the spatio-temporal localization, Monte Carlo simulations have been conducted. The same algorithm is applied at different depths $(Z=5 \mathrm{~mm}, 10 \mathrm{~mm}, 15 \mathrm{~mm}$, $20 \mathrm{~mm}, 25 \mathrm{~mm}$ ), each with 10000 simulated data. For each Monte Carlo run, we consider the following assumptions: 

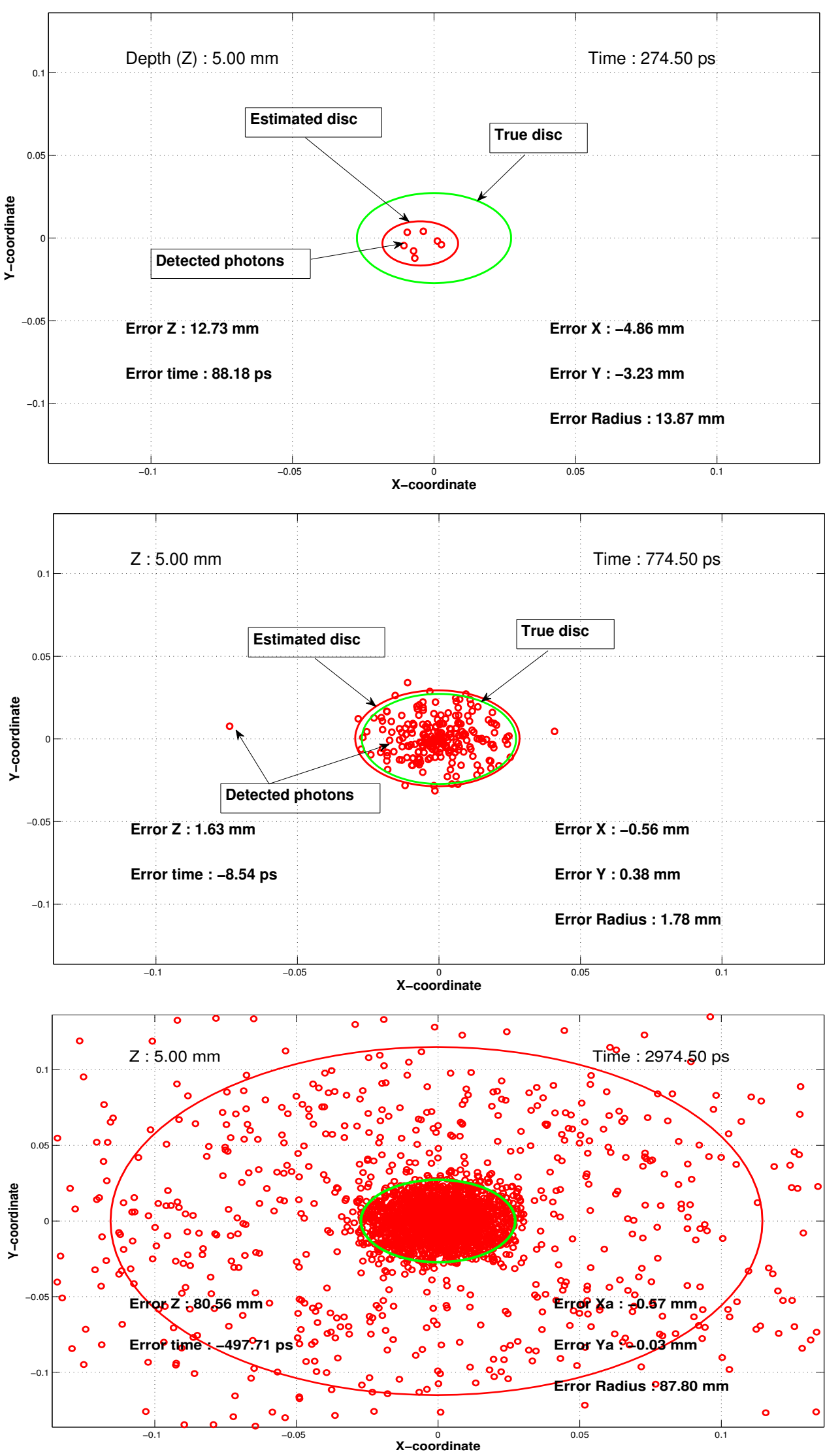

Figure 6. Images at different time shots and corresponding estimation results for $5 \mathrm{~mm}$ depth; true disc is plotted in green and estimated disc in red. 
- The photons' directions, after the scintillation event, are sampled according to a uniform distribution in all 3D directions.

- The detection of un-scattered photons is considered as deterministic. In other words, if incident UV photons have an angle lower than the critical angle $\theta_{c}$, they are considered as detectable by the Si-PM. However, detection efficacy is considered as follows: only a percentage of incident photons are considered as effectively detected. However, this random detection does not affect the property of the spatial distribution of the detected photons: the region inside the critical disc has a higher density than the whole plane.

- The reflection on the lower detector plane is considered as deterministic: the incident photon with an angle higher than $\theta_{c}$ is reflected with the same angle.

- The scattering on the upper plane is considered as random, with a uniform distribution in all 3D directions. A random absorption of the incident photons on the upper surface is also considered.

These Monte-Carlo simulations are implemented with Matlab software, considering simple yet realistic physical assumptions about the scintillation event, the propagation of UV photons, the reflection on the polished surface and the scattering on the upper crystal surface. The Compton effect is not considered in these Monte Carlo simulations. However, as explained above, according to the Klein-Nishina formula, the density of the critical disc is expected to remain higher than the whole plane. The use of the discontinuity of the critical disc is then not affected.

For each depth $Z$, an optimal decision time exists (time when the estimates could be optimally computed). However, as the depth is a priori unknown, one has to set a decision time which will be considered independently of the actual depth. The setting of the decision time should be done while considering the trade-off between 2 aspects: (i) the error on $\mathrm{T}$ and $\mathrm{Z}$ grows over time and (ii) the statistical robustness of the ellipse estimation increases as the number of detected photons increases. Figure 7 shows the total number of detected photons over time for different depths. It is clear that greater depth results in a lower number of photons.

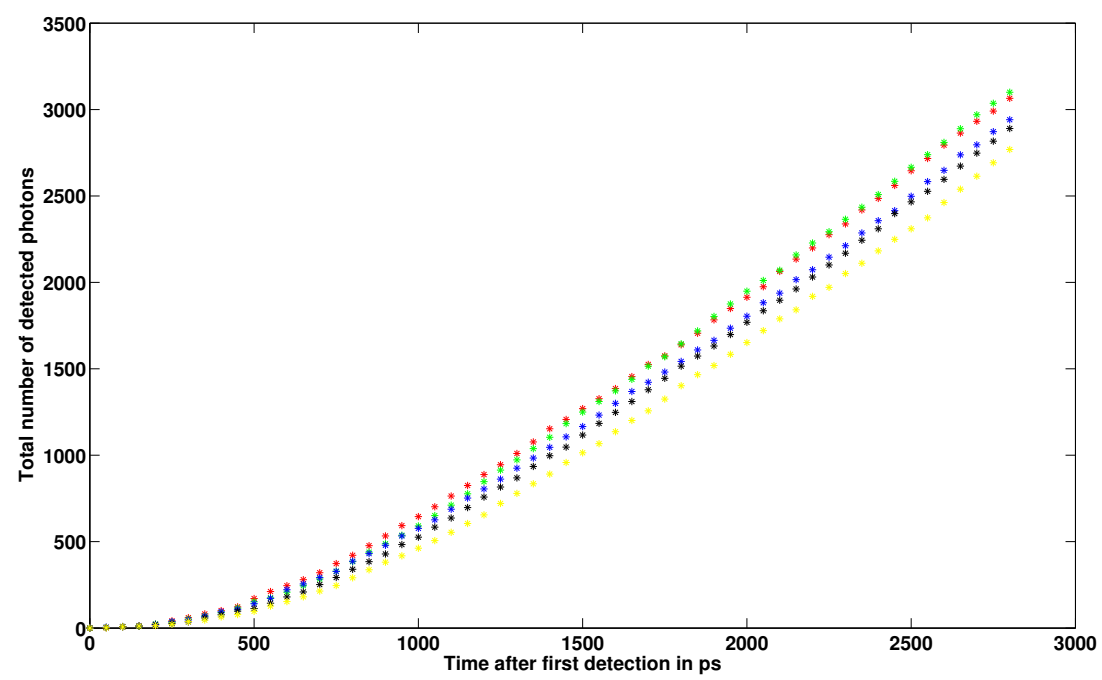

Figure 7. Total number of detected photons for various depths: $5 \mathrm{~mm}$ (red), $10 \mathrm{~mm}$ (blue), $15 \mathrm{~mm}$ (magenta), $20 \mathrm{~mm}$ (black), $25 \mathrm{~mm}$ (cyan). 
On the other hand, Figure 8a shows the evolution of time estimation error with respect to the decision time (Monte Carlo average) and Figure 8b shows the evolution of $\mathrm{Z}$ estimation error with respect to the decision time (Monte Carlo average). It is worth noting that the time error has the same behavior: first, it decreases reaching its minimum (which decreases as $Z$ increases) and then it increases in a monotone way. Consequently, a practical choice of the decision time could be between 500 ps and 750 ps as the number of detected photons is at least 100, which ensures the robustness of the statistical estimation of the critical disc.

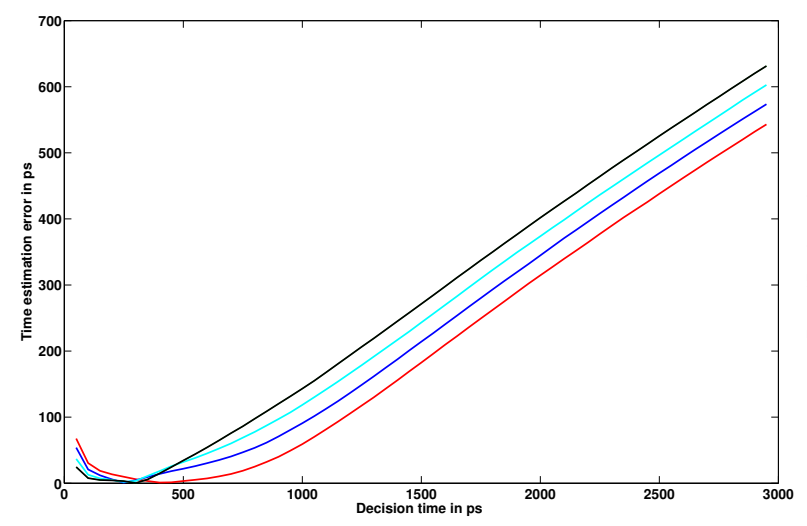

(a)

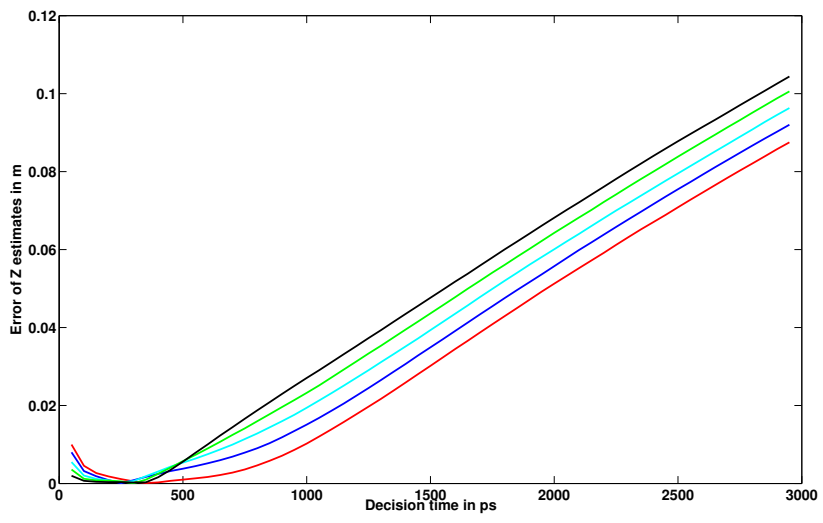

(b)

Figure 8. (a) Absolute error of the $\mathrm{T}$ estimate (in picoseconds) for various depths: $5 \mathrm{~mm}$ (red), $10 \mathrm{~mm}$ (blue), $15 \mathrm{~mm}$ (cyan), $20 \mathrm{~mm}$ (green), $25 \mathrm{~mm}$ (black); (b) Absolute error of the $\mathrm{Z}$ estimate (in meters) for many depths: $5 \mathrm{~mm}$ (red), $10 \mathrm{~mm}$ (blue), $15 \mathrm{~mm}$ (cyan), $20 \mathrm{~mm}$ (green), $25 \mathrm{~mm}$ (black).

In Table 1, the Monte Carlo estimation errors of the spatio-temporal localization are reported for different depths at a decision time $t_{\text {decision }}=700$ ps (after the first photon detection).

Table 1. Monte Carlo averages of absolute errors of $X, Y, Z$ (in $\mathrm{mm}$ ) and $\mathrm{T}$ (in ps) for different depths of the scintillation event. Decision time is taken 700 ps after the first photon detection.

\begin{tabular}{ccccc}
\hline & $\hat{\boldsymbol{X}}_{\mathbf{0}}-\boldsymbol{X}_{\mathbf{0}}^{*}$ & $\hat{\boldsymbol{Y}}_{\mathbf{0}}-\boldsymbol{Y}_{\mathbf{0}}^{*}$ & $\hat{\boldsymbol{Z}}_{\mathbf{0}}-\boldsymbol{Z}_{\mathbf{0}}^{*}$ & $\hat{\boldsymbol{T}}_{\mathbf{0}}-\boldsymbol{T}_{\mathbf{0}}^{*}$ \\
\hline$Z=5 \mathrm{~mm}$ & 0.0042 & 0.01 & 2.8 & 10.3 \\
$Z=10 \mathrm{~mm}$ & 0.0031 & 0.03 & 4 & 35.07 \\
$Z=15 \mathrm{~mm}$ & 0.017 & 0.002 & 6.3 & 52.47 \\
$Z=20 \mathrm{~mm}$ & 0.0142 & 0.02 & 9.3 & 64.72 \\
$Z=25 \mathrm{~mm}$ & 0.0009 & 0.01 & 12 & 75.95 \\
\hline
\end{tabular}

In order to compare the performances of the proposed temporal imager with the state-of-the-art LYSO detectors, Table 2 shows the spatio-temporal localization precision and the energy resolution of both systems. The gain in performances can be seen at all levels: 2D coordinates (X,Y), depth of interaction (Z), timing of interaction $(\mathrm{T})$ and also the energy resolution which can be lower than $5 \%$. In the same table, we have also compared the performances of the proposed temporal imager with two different crystals having different physical properties: $\mathrm{CeBr} 3$ and LYSO. The $\mathrm{CeBr} 3$ crystal shows better results. 
Table 2. Comparison of the proposed temporal imager performances with the state-of-the-art LYSO system.

\begin{tabular}{cccc}
\hline & Today (Pixels LYSO) & Temporal Imager CeBr3 & Temporal Imager LYSO \\
\hline $\mathrm{X}, \mathrm{Y}$ & $5 \mathrm{~mm}$ & $<1 \mathrm{~mm}$ & $<1 \mathrm{~mm}$ \\
$\mathrm{Z}$ & $20 \mathrm{~mm}$ & $<5 \mathrm{~mm}$ & $<5 \mathrm{~mm}$ \\
$\mathrm{~T}$ & $600 \mathrm{ps}$ & $<100 \mathrm{ps}$ & $<150 \mathrm{ps}$ \\
Energy & $12 \%-15 \%$ & $<5 \%$ & $<5 \%$ \\
\hline
\end{tabular}

\section{Practical Aspects}

\subsection{Experimental Aspects}

Designing the proposed temporal imager with present-day electronics will be tricky as the time resolution required from the read-out system is very high. Nevertheless timing rms of 16 ps have been demonstrated with some very fast Asics [5]. A timing resolution of 44 ps has been claimed for the time stamps on digital PMT DPC32000-22-44. It is clear to us that exploiting such an idea for PET requires the development of new electronics, especially the read-out of Si-PMT. The objective of this paper is to demonstrate the performances of the technology in terms of image improvement, so that the development of the necessary electronic can be started. Experimental measurements require a significant effort, in order to avoid any bias or measurement error. They are presently underway.

\subsection{Principle of Read-Out}

In order to analyze the signal properly with a segmented photo-detector (Si-PMT), we need a read-out capable of very rapidly triggering the time of arrival of the first photons on each element of the detector (e.g., each $2 \times 2 \mathrm{~mm}$ square in the example described above). Such an Asic is already available on a 32 channel version [5]. In order to improve the detection quality, it would be interesting to include the number of photons (or charge) collected during a fixed interval (700 ps) by each pixel. Then, for each event, the images would be analyzed to deduce X, Y, Z and T. It is also clear that significant work has to be conducted in the data-processing scheme of such a setup.

\subsection{Count Rates}

In the proposed system, each event can be identified by its spatio-temporal signature:

- Two events quasi-coincident in time but physically distant by more than two plate thicknesses (i.e., $60 \mathrm{~mm}$ ) would be identifiable and could be analyzed separately.

- As long as it is possible to resolve the two discs on a timing image, it should be possible to analyze two events that are spatially close as long as the first event has significantly decayed (2 to $3 \tau=32$ to $48 \mathrm{~ns}$ ). 
Thus, the proposed system should be much less prone to pile-up than conventional monolithic systems (i.e., $\gamma$ cameras).

\section{Conclusions}

This contribution describes a new concept for high spatial and temporal resolution for PET Imaging: the temporal PET camera. This imager is composed of a monolithic plate of fast and high light yield scintillators, such as LaBr3:5\%Ce, coupled with a dense array of fast Si-PMT. The key idea behind this concept is the ability of the system to accurately localize the region of detected un-scattered photons on the Si-PMT. The un-scattered photons are located in a cone whose summit is the interaction position and the opening angle is the critical angle of total reflexion on the lower plate. Those photons arrive first on the detector and the dimension of this cone can be easily measured with proper time gating. This gives the spatial coordinates $(X, Y, Z)$. Ray tracing on the first photons detected thus allows a very precise measurement of timing (T). Our objective in the present paper is to describe the concept and calculate its ultimate performance. Thus, we have not considered limitations related to the size or quantum efficiency of detector elements. If the concept is successful, tailor-made Si-PMT and ASICs to support it will have to be developed. A numerical simulation is provided for a $30 \mathrm{~mm}$ thick plate of $\mathrm{LaBr} 3: 5 \% \mathrm{Ce}$. All the spatial resolution discussion is based on a single event. The precision reached in $\mathrm{X}, \mathrm{Y}$ is sub-millimetric. The precision in $\mathrm{Z}$ varies with depth from 2 to $12 \mathrm{~mm}$. The timing precision is excellent (lower than 80 ps). The best precision in those parameters is reached around 500-700 ps after the event. Thus, even with present-day electronics performance, it should be possible to reach good spatial resolution with such a system. The concept should therefore allow us to build PET with vastly improved spatial and timing resolutions. In addition, the equipment could be significantly cheaper to produce in large quantities than today's LSO PET systems.

\section{Author Contributions}

Both authors contributed to this research work. Alain Iltis is in charge of the physics and Hichem Snoussi in charge of simulation and data processing. Both authors contributed to the writing of the manuscript.

\section{Conflicts of Interest}

The authors declare no conflict of interest.

\section{References}

1. Seifert, S.; van Dam, H.T.; Huizenga, J.; Vinke, R.; Dendooven, P.; Lohner, H.; Schaart, D.R. Monolithic LaBr3: Ce crystals on silicon photomultiplier arrays for time-of-flight positron emission tomography. Phys. Med. Biol. 2012, 57, 2219-2233.

2. Anger, H.O. Gamma-Ray and Positron Scintillation Camera. Nucleonics Ceased Publication 1963, 21, UCRL-10933. 
3. Pani, R.; Vittorini, F.; Pellegrini, R.; Bennati, P.; Cinti, M.N.; Mattioli, M.; de Notaristefani, F. High spatial and energy resolution gamma imaging based on $\mathrm{LaBr} 3(\mathrm{Ce})$ continuous crystals. In Proceedings of the Nuclear Science Symposium Conference Record (NSS'08), Dresden, Germany, 19-25 October 2008; pp. 1763-1771.

4. Iltis, A.; Mayhugh, M.R.; Menge, P.; Rozsa, C.M.; Selles, O. Solovyev, V. Lanthanum halide scintillators: Properties and applications. Nucl. Instrum. Methods Phys. Res. Sect. A: Accel. Spectrom. Detect. Assoc. Equip. 2006, 563, 359-363.

5. Fleury, J. Petiroc and Citiroc: Front-end ASICs for SiPM read-out and ToF applications. JINST 2014, 9, C01049

6. Lewellen, T.K.; Murano, R. A comparison of count rate parameters in gamma cameras. J. Nucl. Med. 1981, 22, 161-168.

7. Adam, L.E.; Karp, J.S.; Daube-Witherspoon, M.E.; Smith, R.J. Performance of a whole-body PET scanner using curve-plate NaI(Tl) detectors. J. Nucl. Med. 2001, 42, 1821-1830.

8. Iltis, A. Système et Procédé de Détection de Rayonnement Gamma de Type Gamma camera, Patent FR 1260596, 8 November 2012.

9. Yamamoto, S. Development of Si-PM Based Imaging Systems for Molecular Imaging. J. Med. Bioeng. 2013, 2, doi: 10.12720/jomb.2.1.16-19

10. Jarritt, P.H.; Acton, P.D. PET imaging using gamma camera systems: A review. Nucl. Med. Commun. 1996, 17, 758-766.

11. Bourgeois, C. Interaction particules-matière: Théorie; Techniques de l'ingénieur: Paris, France, 1998; AF3 530.

12. Gonzalez Martinez, A.J.; PeirÃş Cloquell, A.; SÃąnchez Martinez, F.; Vidal San Sebastian, L.F.; Benlloch Baviera, J.M. Innovative PET detector concept using Si-PMT and continuous crystals. Nucl. Instrum. Methods Phys. Res. Sect. A 2012, 695, 213-217.

13. Klein, O.; Nishina, Y. Über die Streuung von Strahlung durch freie Elektronen nach der neuen relativistischen Quantendynamik von Dirac. Z. Phys. 1929, 52, 853-869.

(C) 2015 by the authors; licensee MDPI, Basel, Switzerland. This article is an open access article distributed under the terms and conditions of the Creative Commons Attribution license (http://creativecommons.org/licenses/by/4.0/). 